\title{
A REAL-TIME LOCATION-BASED CONSTRUCTION LABOR SAFETY MANAGEMENT SYSTEM
}

\author{
Kwang-Pyo LEE ${ }^{\mathrm{a}}$, Hyun-Soo LEE ${ }^{\mathrm{a}}$, Moonseo PARK ${ }^{\mathrm{a}}$, Hyunsoo KIM ${ }^{\mathrm{a}}$, Sangwon HAN ${ }^{\mathrm{b}}$ \\ ${ }^{a}$ Department of Architecture, Seoul National University, Seoul, Korea \\ ${ }^{\mathrm{b}}$ Department of Architectural Engineering, University of Seoul, Seoul, Korea \\ Received 24 May 2012; accepted 28 Aug 2012
}

\begin{abstract}
The construction industry continues to record a high number of accidents compared to other industries. Furthermore, the ramifications of construction accidents are growing in terms of both economic loss and loss of life with trends toward larger-scale, more complex projects. For this reason, there is an increasing awareness of the importance of safety management in the construction industry, and the need for more effective safety management techniques. This paper introduces a real-time location-based construction labor safety management system that tracks and visualizes workers' locations in real-time and sends early warnings to endangered workers. The system is developed by integrating: a realtime locating system (RTLS) for tracking of workers' location; a location monitoring system for mapping the workers location on a computerized building model; and alarm technology for sending early warnings. The developed system has been applied to an apartment project and an RTLS technology test center in Korea, and proved to be effective in tracking and monitoring workers in real-time and preventing construction accidents. It is envisioned that the developed system will enable proactive construction safety management in South Korea and the methodologies developed in this study will be applicable to other contexts with minimal customization.
\end{abstract}

Keywords: construction safety, real-time locating system (RTLS), radio frequency identification, alarm technology, tracking, risk assessment, South Korea.

\section{Introduction}

The construction industry continues to record a high incidence of accidents compared to other industries. Recent records indicate that on average, $1.24 \%$ of construction employees have faced a calamity or suffered an accident while working. This number is much higher than in other industries, including transportation and warehousing $(0.86 \%)$, manufacturing $(0.46 \%)$, and mining $(0.19 \%)$ (US Bureau of Labor 2006). With trends toward larger-scale and more complex construction projects, the ramifications of construction accidents are growing in terms of both economic loss, loss of life as well as social impacts such as the incapacity to work due to an injury. This highlights the importance of safety management in construction industry and supports a need for a more effective construction safety management system (Hinze et al. 1998).

To find an effective method for the prevention of construction accidents, an understanding of the mechanism of accidents generation is required. According to Heinrich (1959), every accident has its causes and can be prevented by identifying and eliminating the causes. Heinrich (1959) also classified causes of an accident into direct and indirect causes of accidents, where indirect causes are 'deficiency in safety management' and direct causes include 'unsafe mechanical or physical conditions' and 'unsafe acts of a person'. This suggests that a lack of safety management effort can result in either unsafe working conditions or unsafe acts, or both of them. Some combinations of these direct causes eventually trigger accidents. This means that an accident can be prevented or at least reduced by removing either unsafe conditions or unsafe acts.

In an effort to reduce accidents, diverse safety management activities have been implemented across the construction industry, including the Construction Safety and Health Program (CSHP) and the Construction Accident Prevention Technique (CAPT) (Reese, Eidson 2006). These safety management efforts, however, have mainly focused on the indirect causes of construction accidents (i.e. deficiency in safety management) without due concentration on the direct causes, which can vary significantly for each construction site depending on its given circumstance (Navon, Kolton 2007). For this reason, current safety management efforts usually provide general suggestions for preventing accidents. Detailed information is scarcely provided regarding the specific accident types expected on a particular construction site, and actions that would be effective to prevent them.

For effective prevention of construction accidents, both direct and indirect causes of accidents should be analyzed simultaneously. As an effort to address this necessity, diverse research efforts have been conducted on visualization or location-based safety management (Teizer, Castro-Lacouture 2007; Chae, Yoshida 2010; 
Carbonari et al. 2011; Chi, Caldas 2012; Lee et al. 2012). For example, Carbonari et al. (2011) developed a prototype for proactive construction safety management and real-time signaling of potential overhead hazards. Also, Lee et al. (2012) developed radio frequency identification (RFID)-based real-time locating system (RTLS) for construction safety management. In addition, $\mathrm{Chi}$ and Caldas (2012) presented an automated image-based safety assessment method for earthmoving and surface mining activities.

Motivated by these research efforts, this paper aims to develop a construction safety management system by which rigor around monitoring of direct accident causes can be improved, particularly tracking and visualizing workers' location in a real-time and proactive manner. This paper achieves the research objective through integrating: real-time locating system (RTLS) for tracking of workers' locations; a location monitoring system using ArchiCAD 12 for mapping workers' movement on a computerized building model and processing workers' real-time location data; and alarming technology for sending early warnings including detailed information to endangered workers. Also, risk assessment is conducted to identity potential dangerous zones and their associated hazard types.

This paper is presented in the following order: an overview of related methodology and technologies is provided; risk assessment is conducted based on historical construction accident database in South Korea; system modules are discussed, including RTLS, location monitoring system, alarming technology, and data protocol. Then, two case studies are conducted to test the location tracking and mapping performance of the developed system. The final section provides the conclusions and future directions.

\section{Preliminary study}

Prior to development of the construction safety management system, this section briefly reviews relevant works, particularly those related to visualization and tracking technologies for construction performance monitoring purposes.

\subsection{Visualization for monitoring}

Visualization methods for monitoring are classified into two major categories: Virtual Reality (VR) and Augmented Reality (AR). VR aims to exactly reproduce realworld situations as a computer simulation, to predict how the project will progress. In this way, VR can identify potential problems during building construction. For example, Issa (2000) introduced VR as a platform to integrate various data and applications. Heesom et al. (2003) developed a dynamic VR system, making it possible to derive an effective layout for space usage on construction sites. Irizarry and Abraham (2005) presented a VR-based methodology to improve safety in steel erection. On the other hand, AR superimposes virtual images created by computing onto real images (e.g. a photo), and identifies differences between the two. Relating to this, GolparvarFard et al. (2009) introduces an advanced approach that uses photographs to generate point clouds which are compared to the BIM geometry model for progress monitoring, and progress discrepancies through color and pattern using this innovative simulation model. Behzadan and Kamat (2009) developed a methodology to generate smooth and continuous AR animations from the results of discrete event simulation models. Dai et al. (2011) suggested an analytical approach to incorporating computergenerated 3D graphic of invisible underground infrastructure into the construction site so as to present an integral view of the site situation in construction engineering application.

\subsection{Location tracking for monitoring}

The discipline of construction progress monitoring has shifted from paper-based data collection and analysis, which is time-consuming and error-prone, to automated data collection. Initially, the bar code technique was used for automated data collection until its low level of recognition and insufficient storage capacity resulted in its replacement by the Radio Frequency Identification (RFID) technique (Ergen e al. 2007). RFID, however, is limited for portal based identification purposes: thus, the need for a more advanced type of RFID has arisen (Song et al. 2006). To address this need, there have been numerous research efforts for the advanced real-time locating system (RTLS) for diverse purposes in construction (e.g. safety management, productivity improvement and material tracking) (Fosbroke 2004; Teizer, CastroLacouture 2007; Wu et al. 2010; Chae, Yoshida 2010; Cheng, Teizer 2010). These studies can be largely categorized into: 1) the application of RFID to whole parts of the construction site and its procedures; and 2) developing an algorithm for improving feasibility of localization. As an effort to apply RFID to a whole construction site, Jaselskis and El-Misalami (2003) proposed the application of a material tracking system considering cost, schedule, quality and safety of the whole project process, and conducted a pilot test. Chin et al. (2008) suggested a management system that monitors progress on structural steel erection using RFID and 4D CAD. Also, for improving performance of localization, Song et al. (2006) proposed the proximity method that allows tracking of material effectively on a construction site. Dziadak et al. (2009) developed a model to manage the location of buried assets using RFID. Jing et al. (2008) suggested a method using RFID and two-dimensional code technology to manage people flow and logistics more effectively.

\subsection{Tracking and visualization technologies for construction safety management}

There have been significant research efforts applying location tracking or visualization technology for a systematic construction safety management (Teizer, CastroLacouture 2007; Fullerton et al. 2009; Hallowell et al. 2010; Chae, Yoshida 2010; Chi, Caldas 2011). These efforts focused on directly managing workers and objects to improve safety performance by proactively capturing site information (Lee et al. 2012). Fullerton et al. (2009) 
developed pro-active-real-time safety technology using radio frequency wave spectrum to improve the safety of a work zone by alerting workers. Hallowell et al. (2010) identified the potential barriers and enablers associated with integrating sensing technologies within existing safety management strategies by interviewing safety managers. Chae and Yoshida (2010) applied Radio Frequency Identification (RFID) technology for prevention of collision accidents with heavy equipment. Teizer and Castro-Lacouture (2007) introduced an approach leveraging the advancements of wireless Ultra-Wideband (UWB) positioning and reflectorless Range Imaging (RIM) sensing technology in the construction productivity and safety monitoring. Chi and Caldas (2011) presented an automated object identification method using standard video cameras on construction sites.

\section{Risk assessment by work type}

In order to develop an effective real-time construction safety management system, construction safety risk needs to be assessed for identifying potential dangerous zones. Construction safety risk is used to measure the extent of risk in a given dangerous area. In order to assess the extent of risk for a specific work type, we utilize the Korea Occupational Safety and Health Agency (KOSHA)'s 2007 report, which is one of the most comprehensive risk assessment models in the Korean construction industry. KOSHA's 2007 report summarizes the analysis on about 18,000 accident cases which were occurred in 2006.

According to the KOSHA'S 2007 report, the risk assessment by work type consists of three steps: hazard identification, risk calculation, and risk assessment. Hazard identification is performed in two stages: 1) work type selection; and 2) hazard identification. In the work type selection stage, the work type to be assessed is selected and the scope of assessment is determined. The work type is composed by activities and the activities are analyzed using the previous accident cases for assessing the level of danger. Hazards are identified based on unsafe factors like unsafe behavior of worker, unsafe materials and substances, unsafe working method, and unsafe equipment. In the risk calculation step, the risk is calculated by multiplying degree of severity and frequency of a work type. The frequency is calculated based on total number of injured workers and the number of injured workers per work type. Also, the severity of risk is calculated based on the number of injured workers per work type and a conversion value from the loss of workdays by work type. The severity and frequency of risk is calculated according to the equations below:

Frequency $=\frac{\text { Injured worker's number by work type }}{\text { Total number of injured worker }} \times 100$;

Severity $=\frac{\text { Conversion value from loss of workday by work type }}{\text { Injured worker's number of work type }}$.

Table 1 shows an example of risk assessment by work type for apartment construction. According to the KOSHA's 2007 report, apartment construction is classi- fied into 32 work types (e.g. foundation, excavation, blasting, retaining wall bearing, refilling, form work, and so on) and the risk of each work type is calculated. Using this data, we classified the work types to five grades from 1 (low risk) to 5 (high risk) depending on the degree of risk by work type. Then, the degree of risk is visualized by five colors (i.e. red $=5$, orange $=4$, yellow $=3$, green $=2$, and blue $=1$ ).

Table 1. Risk Assessment by work type

\begin{tabular}{|c|c|c|}
\hline Work type & Risk & Grade and color \\
\hline Form work & 24.259 & $\begin{array}{c}5 \text { grade represented } \\
\text { by red color }\end{array}$ \\
\hline Machinery & 14.657 & \multirow{5}{*}{$\begin{array}{l}4 \text { grade represented } \\
\text { by orange color }\end{array}$} \\
\hline Interior finishing & 11.955 & \\
\hline Electric installation & 10.780 & \\
\hline Plastering & 9.414 & \\
\hline Panel & 8.295 & \\
\hline Reinforced & 6.665 & \multirow{7}{*}{$\begin{array}{l}3 \text { grade represented } \\
\text { by yellow color }\end{array}$} \\
\hline Manhole & 5.878 & \\
\hline Earth & 5.270 & \\
\hline Steel-frame & 4.848 & \\
\hline Watertight & 4.756 & \\
\hline Painting & 4.553 & \\
\hline Safety scaffolding & 4.016 & \\
\hline Tile & 3.541 & \multirow{6}{*}{$\begin{array}{l}2 \text { grade represented } \\
\text { by green color }\end{array}$} \\
\hline Window \& Door & 3.437 & \\
\hline Concrete & 3.265 & \\
\hline Metal & 2.184 & \\
\hline Landscaping & 2.142 & \\
\hline Retaining wall bearing & 1.395 & \\
\hline $\mathrm{E} / \mathrm{V}$ & 0.630 & \multirow{13}{*}{$\begin{array}{l}1 \text { grade represented } \\
\text { by blue color }\end{array}$} \\
\hline Excavation & 0.573 & \\
\hline Gang-form work & 0.482 & \\
\hline Working environement & 0.411 & \\
\hline Tower crane & 0.397 & \\
\hline Foundation & 0.366 & \\
\hline Grouting & 0.344 & \\
\hline Temporary road & 0.321 & \\
\hline Refilling & 0.273 & \\
\hline Blasting & 0.153 & \\
\hline Shifting inconvenience & 0.053 & \\
\hline Temporary electrictity & - & \\
\hline Danger equipement & - & \\
\hline
\end{tabular}

Based on this, the safety manager can identify the dangerous zone and potential accident types according to construction schedule. Potential accidents types are classified based on the Hinze and Russell's classification, which consists of 14 different types of accident including falls, dropping, collapse, strangulation, electric shock, landslide, collision, suffocation, fire, overturning, destruction, explosion, and drowning. Particularly, in the South Korean construction industry, falls, electric shock, dropping, collision, and strangulation were identified as the major accident types. This analysis result is not very different from studies conducted in other countries which can expect the applicability of the developed system to other contexts. 


\section{System development}

This section provides a system overview and a detailed explanation of the system modules and data transmission protocol among the modules. The modules include realtime locating system, location monitoring system and alarming technology.

\subsection{System overview}

The proposed construction safety management system tracks workers' location in a real-time manner by applying a Real-Time Locating System (RLTS), and visualizes workers' locations using a location monitoring system developed based on ArchiCAD 12 (GRAPHISOFT 2008). When a worker approaches a dangerous area, it automatically (or manually) sends warning signal to the worker through alarm technology (Fig. 1). By doing so, this system can assist to minimize unsafe conditions (i.e. direct cause of accidents), thereby preventing accidents in a construction site. In order to identify dangerous situations and hazard types for a given work type, risk assessment is conducted as described in the previous section.

\subsection{Real time locating system (RTLS) based tracking}

For the purpose of tracking the real-time location of workers, a RTLS system is developed. This section describes the RTLS hardware configuration and localization methods.

\section{1) Hardware configuration}

The RTLS hardware is composed of four main parts: tag, reader, base-station and engine. A tag is attached to each worker to be tracked. Each tag measures its distance from surrounding readers and then transmits this reader-to-tag distance data to the base-station. A reader is classified as either stationary or portable, both of which are used in this system. A stationary reader is utilized where power can be provided at its installation location, and a portable reader is utilized otherwise. The base- station receives the reader-to-tag distance data and then processes and sends the data to the engine for localization. Finally, the RTLS engine functions as the main server for the system, handles the data processing and storage for output, and filters the data it receives from the base station to maximize the tracking performance. Table 2 shows the functions and specifications of the RTLS hardware.

\section{2) Localization methods}

The RTLS localization methods consist of position determination method and wireless networking method. The position determination method is applied to identify a worker's location and the wireless networking method is utilized to support data communication between the hardware components. Several techniques are being utilized for the position determination including Received Signal Strength Indication (RSSI), Time Difference of Arrival (TDOA), Time of Flight (TOF), Angle of Arrival (AOA) and Time of Arrival (TOA). Table 3 summarizes the position determination methods.

While RSSI, TDOA, and TOF are being widely utilized for tracking purposes, they are not suitable in construction environments. RSSI, for example, can encounter a high level of localization error due to the multipath problem where obstacles are prevalent. TDOA can maintain high performance in location tracking in a clear Line Of Sight (LOS) environment which is unusual in construction. Also, TDOA and TOF require time synchronization (consistency of reader and tag time) which can make their application in construction difficult. The AOA technique requires an isotropic antenna which has limited communication range. TOA also requires time synchronization, but is more stable compared to TDOA or TOF method. Moreover, TOA is highly robust in tracking object positions and has a large coverage area without being largely affected by the multipath problem. TOA has therefore been chosen for this research.

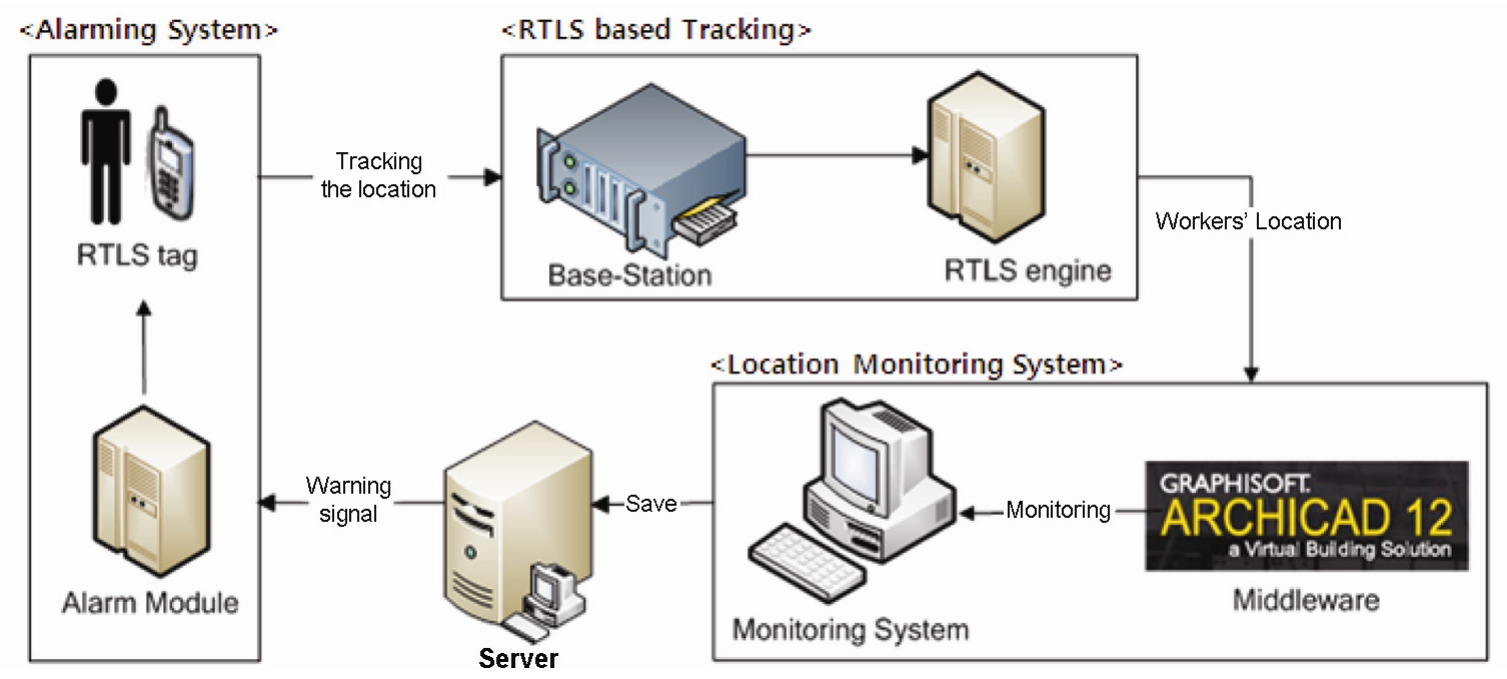

Fig. 1. System architecture 
Table 2. RTLS hardware

\begin{tabular}{|c|c|c|c|}
\hline Classification & Function & Specifications & Figure \\
\hline Tag & $\begin{array}{l}\text { - Tracks a worker's location us- } \\
\text { ing receptions from reader }\end{array}$ & $\begin{array}{l}\text { - Processor: TI MSP430 } \\
\text { - RF Transceiver: NA5TR1 } \\
\text { - Transmission distance: } 450 \mathrm{~m}\end{array}$ & \\
\hline Reader & $\begin{array}{l}\text { - Works as a reference point to } \\
\text { identify location of a tag }\end{array}$ & $\begin{array}{l}\text { - ARM co.'s Cortex-M3 } \\
\text { - } 72 \mathrm{MHz} \text { Movement } \\
\text { - } 128 \mathrm{~KB} \text { flash, } 20 \mathrm{~KB} \text { RAM }\end{array}$ & \\
\hline Base-Station & $\begin{array}{l}\text { - Receives distance data from the } \\
\text { tag and passes it to the engine }\end{array}$ & $\begin{array}{l}\text { - } 400 \mathrm{MHZ} \text { Intel PXZ255 MCU } \\
\text { - 10/100 Mbps TCP/IP compatible }\end{array}$ & \\
\hline \multirow{3}{*}{ Engine } & \multirow{3}{*}{$\begin{array}{l}\text { - Filters and processes distance } \\
\text { data from the base station }\end{array}$} & \multirow{3}{*}{$\begin{array}{l}\text { - Multi-Lateration: Trilateration } \\
\text { - Data filtering: Statistical method }\end{array}$} & $=$ \\
\hline & & & 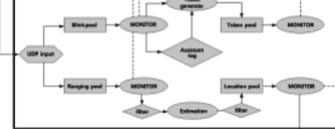 \\
\hline & & & \\
\hline
\end{tabular}

Table 3. Position determination method (based on Bahl and Padmanahan 2000)

\begin{tabular}{|c|c|c|c|}
\hline Techniques & Position determination method & Merit & Demerit \\
\hline $\begin{array}{l}\text { Received Signal } \\
\text { Strength } \\
\text { Indication } \\
\text { (RSSI) }\end{array}$ & $\begin{array}{l}\text { - Calculates the location using } \\
\text { signal strength from access point }\end{array}$ & $\begin{array}{l}\text { - Hardware is realized relatively } \\
\text { cheaply and easily }\end{array}$ & $\begin{array}{l}\text { - Influenced severely by } \\
\text { multipath } \\
\text { - Accuracy is affected by } \\
\text { distance }\end{array}$ \\
\hline $\begin{array}{l}\text { Time Difference } \\
\text { of Arrival } \\
\text { (TDOA) }\end{array}$ & $\begin{array}{l}\text { - Similar to TOA but calculates us- } \\
\text { ing signaling intervals between } \\
\text { readers }\end{array}$ & $\begin{array}{l}\text { - Accepts TOA merits } \\
\text { - Differs from TOA because TDOA } \\
\text { is not depend on the clock bias of } \\
\text { the transmission node } \\
\text { - Performs well in a NLOS } \\
\text { environment as well as outside }\end{array}$ & $\begin{array}{l}\text { - Needs partial time } \\
\text { synchronization }\end{array}$ \\
\hline $\begin{array}{l}\text { Time of Flight } \\
\text { (TOF) }\end{array}$ & $\begin{array}{l}\text { - Uses the time elapsed when the } \\
\text { signal is transferred between the } \\
\text { tag and reader, based on the trans- } \\
\text { fer rate of the RF signal }\end{array}$ & $\begin{array}{l}\text { - Can extract results relatively } \\
\text { easily and quickly }\end{array}$ & $\begin{array}{l}\text { - Needs time } \\
\text { synchronization }\end{array}$ \\
\hline $\begin{array}{l}\text { Angle of Arrival } \\
\text { (AOA) }\end{array}$ & $\begin{array}{l}\text { - Location calculated using distance } \\
\text { and position of RF sign signaling } \\
\text { tag and receiving reader }\end{array}$ & $\begin{array}{l}\text { - Accuracy is not affected by } \\
\text { distance } \\
\text { - Minimal influence due to } \\
\text { multipath }\end{array}$ & $\begin{array}{l}\text { - Needs an isotropic } \\
\text { antenna and antenna } \\
\text { array } \\
\text { - Cannot extract exact } \\
\text { location coordinates }\end{array}$ \\
\hline $\begin{array}{l}\text { Time of Arrival } \\
\text { (TOA) }\end{array}$ & $\begin{array}{l}\text { - Estimates distance between reader } \\
\text { and tag using } R F \text { signaling speed }\end{array}$ & $\begin{array}{l}\text { - Its accuracy is not affected by } \\
\text { distance } \\
\text { - Minimal influence due to } \\
\text { multipath }\end{array}$ & $\begin{array}{l}\text { - Needs partial time } \\
\text { synchronization }\end{array}$ \\
\hline
\end{tabular}


The TOA technique uses a trilateration technique based on multilateration (Fig. 2). First, based on the reference coordinates $(\mathrm{x}, \mathrm{y})$ of the readers, the distance between the tag and each respective reader is calculated using the time of signal arrivals between the readers and the tag. Pending the availability of distance data from at least three readers per tag, the coordinate of the tag is derived through the trilateration technique.

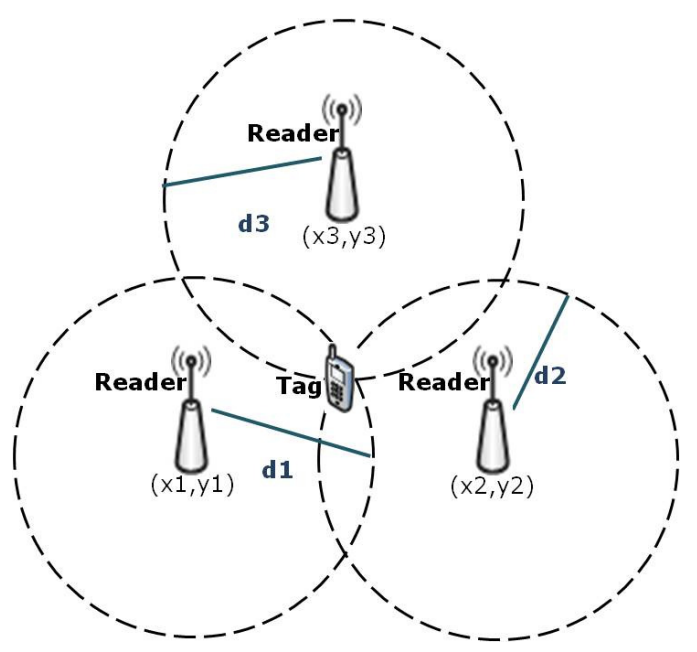

Fig. 2. Multi-lateration (TOA)

The wireless networking technique is required to send the distance data to a base station or engine. There are several networking techniques, as shown in Table 4. The Chirp Spread Spectrum (CSS) technique has been adopted in this research. The CSS works well to mitigate multipath fading and channel noise, and enables precision ranging with a long range of measurement by comparison to other techniques (Son et al. 2009). In CSS, fast data transmission is available on $2.45 \mathrm{GHz}$ and its speed can reach $2 \mathrm{Mb} / \mathrm{s}$. The signal transmission distance is $1 \mathrm{~km}$, which is enough to cover a large-scale building project. The CSS method has an expanded range of data communication and a high level of tolerance for both multiplicity and complexity that can result from the overloading and overlapping of passages and portals. In addition, the CSS is compatible with other networking methods, which reduces the chance of a signal disturbance and minimizes the potential error in location coordinate determination. Using TOA and CSS, information concerning the tag's position is derived and transferred to the RTLS engine in the format of $\mathrm{x}$ and $\mathrm{y}$ coordinates and floor information (i.e. $\mathrm{z}$ coordinate).

\subsection{Location monitoring system}

In order to visualize the movement of workers transmitted by RTLS, a location monitoring system was developed based on ArchiCAD 12 environments (Fig. 3). The monitoring system should seamlessly receive the realtime location data, and process the data within the monitoring process for the purpose of increasing the scalability of system. For this, the monitoring system allows the system users to identify the location of workers and designate the dangerous zone in the building. The monitoring system has four main functions: 1) registering the building; 2) designation of dangerous zones; 3 ) establishing reader reference points; and 4) establishing reader locations.

The building registration function enables the users to represent the outline of a building in the monitoring system. To register the building, it is important to set up a reference point for the building in the location monitoring system. Once the user designates the global reference point of the building in the system, the building is set to measure its coordinates from the reference point. After this, the user chooses the polyline element and draws the outline of building, which is extracted by floors and is stored on the RTLS server.

Designation of dangerous zone function allows the user to specify the dangerous zones within the building by using the fill element provided in ArchiCAD 12 library. The dangerous zone is set by the construction safety manager and the alarm for the potential accident types in a given zone is sent to the worker in real-time manner. In addition, the safety manager can set a threshold value for sending the early alarming to the worker by considering

Table 4. Comparison of wireless networking methods

\begin{tabular}{l|c|c|c|c|c|l}
\hline & RF range & $\begin{array}{c}\text { Transmission } \\
\text { velocity }\end{array}$ & $\begin{array}{c}\text { Transmission } \\
\text { distance }\end{array}$ & Electric power & Net organization & Standard association \\
\hline $\begin{array}{l}\text { Wireless Local Area } \\
\begin{array}{l}\text { Network } \\
\text { (Wireless LAN) }\end{array}\end{array}$ & $2.4 / 5 \mathrm{GHz}$ & $11 \sim 54 \mathrm{Mbp} / \mathrm{s}$ & $50 \mathrm{~m}$ & $800 \sim 1,600 \mathrm{~mW}$ & P2P, Star & $\begin{array}{l}\text { IEEE } 802.11 \\
\text { WiFi } \\
\text { Alliance }\end{array}$ \\
\hline Bluetooth & $2.4 \mathrm{GHz}$ & $1 \mathrm{Mbp} / \mathrm{s}$ & $10 \mathrm{~m}$ & $50 / 80 \mathrm{~mW}$ & $\begin{array}{c}\text { P2P, Star, } \\
\text { Ad-hoc }\end{array}$ & $\begin{array}{l}\text { IEEE } 802.15 .1 \\
\text { Bluetooth } \\
\text { SIG }\end{array}$ \\
\hline $\begin{array}{l}\text { ZigBee } \\
\begin{array}{l}868 / 915 \mathrm{MHz} \\
2.4 \mathrm{GHz}\end{array}\end{array}$ & $250 \mathrm{Kbp} / \mathrm{s}$ & $10 \sim 75 \mathrm{~m}$ & $1 / 75 \mathrm{~mW}$ & $\begin{array}{c}\text { P2P, Star, } \\
\text { Mesh }\end{array}$ & $\begin{array}{l}\text { IEEE } 802.15 .4 \\
\text { Zigbee } \\
\text { Alliance }\end{array}$ \\
\hline $\begin{array}{l}\text { Ultra-Wide Bandwidth } \\
\text { (UWB) }\end{array}$ & $\begin{array}{c}3.1 \sim 10.6 \mathrm{GHz} \\
\begin{array}{l}\text { Chirp Spread Spectrum } \\
\text { (CSS) }\end{array}\end{array}$ & $480 \mathrm{Mbp} / \mathrm{s}$ & $20 \mathrm{~m}$ & $\sim 200 \mathrm{mw}$ & P2P, Mesh & $\begin{array}{l}\text { IEEE } 802.15 .3 \mathrm{a} \\
\text { WiMedia }\end{array}$ \\
\hline
\end{tabular}




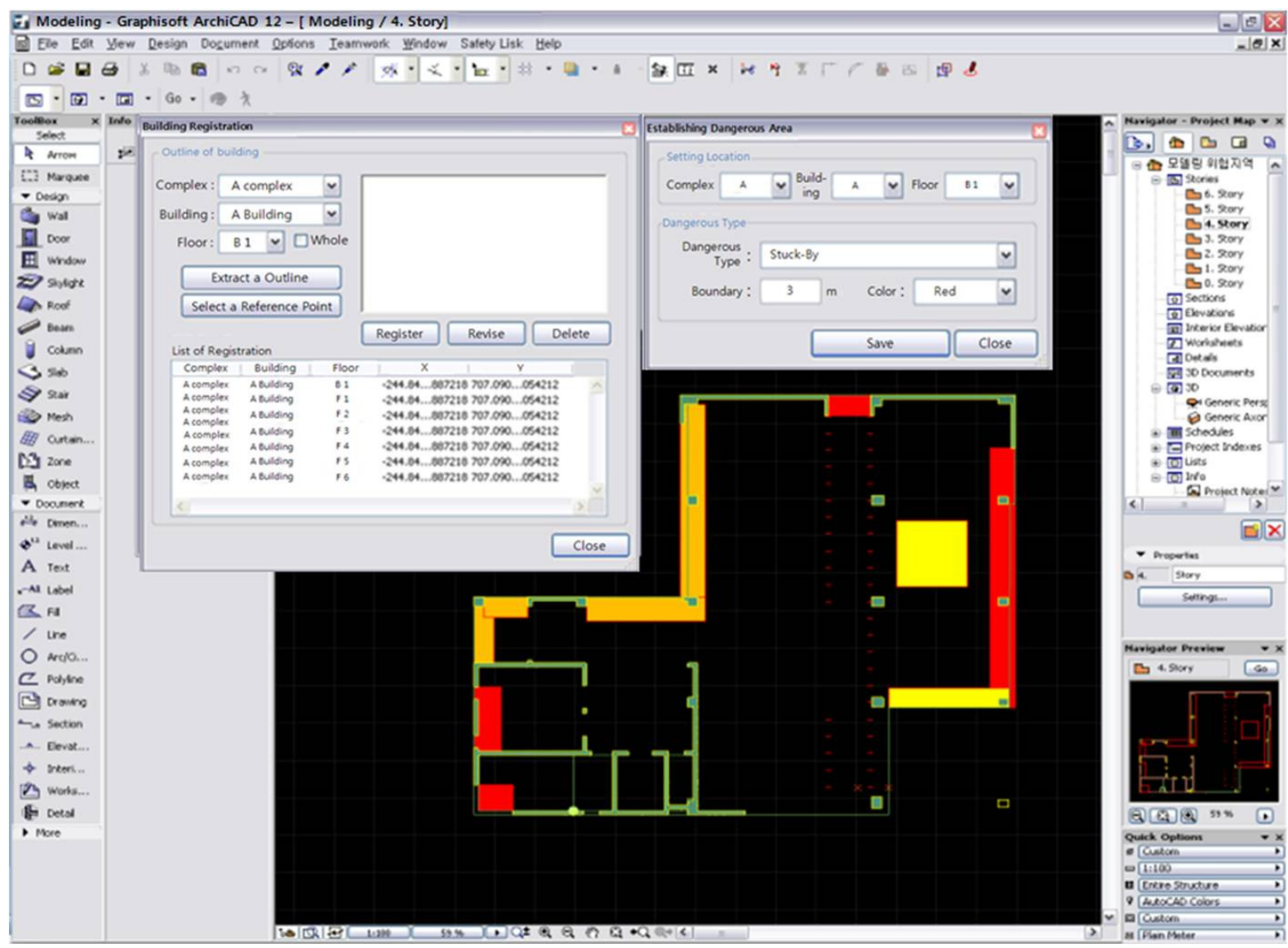

Fig. 3. Location monitoring system

the performance of tracking when the worker is in the vicinity of the dangerous zone. In this research, the threshold was set to be $1.5 \mathrm{~m} / \mathrm{s}$ considering the average movement speed of an adult male (Tarawneh 2001). Further research effort on the optimal size of the threshold is required by carefully analyzing the moving velocity of a worker or object in construction sites and the reaction time of the worker.

The establishment of readers' reference point function sets a reference point for setting the coordinates of the readers. Using this function, the user can set the reference point of readers in the location monitoring system and store the coordinate in the RTLS server. Lastly, reader's locations input function is used to determine the location of readers. Using this function, the user sets the location of the reader and inputs the specification of reader, building, and floor.

\subsection{Alarming system}

Alarm technology is applied to send warning signals to alert a worker when he or she approaches a dangerous area (i.e. unsafe condition). The alarming system is composed of an alarm transmitter and a receiver. The transmitter receives alarm signals from the RTLS engine and delivers it to the alarm receiver, which then sends it to the worker on site. The alarm transmitter and the receiver are attached to the RTLS engine and each tag respectively, as these attachments are highly suitable for use on work sites. Once a worker's location coordinates are sent to the
RTLS engine, the engine compares the coordinates of dangerous areas (specified in the location monitoring system) with the worker location coordinates (transferred from the RTLS).

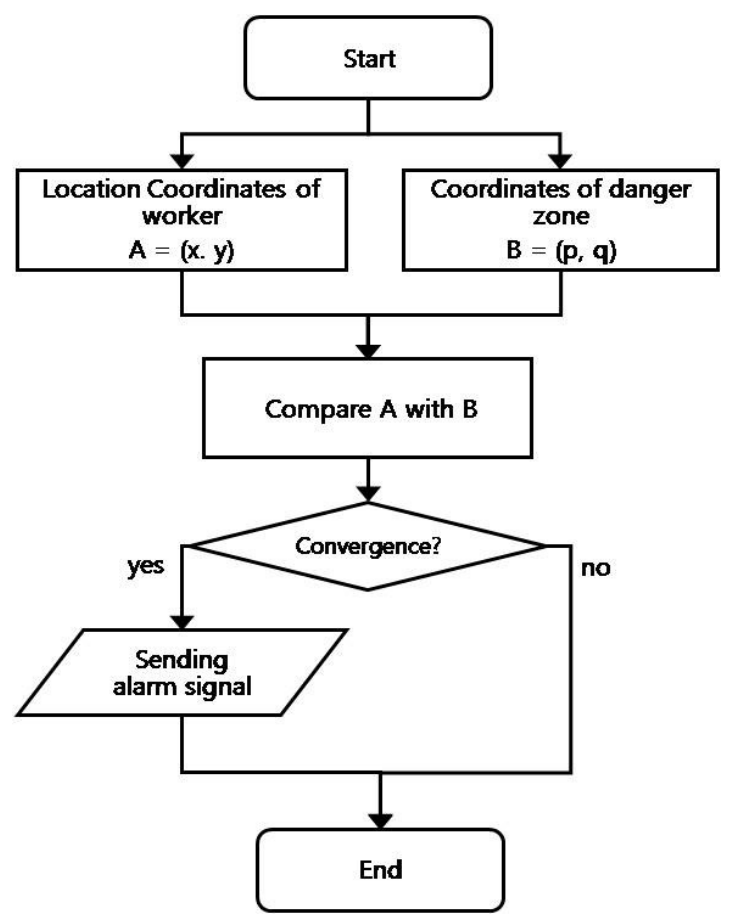

Fig. 4. Alarm signal transmission algorithm 
Figure 4 shows the alarm signal transmission algorithm. The principle of this algorithm is to confirm whether there is an overlap between the coordinates of a worker location and those of dangerous areas. The location coordinate of a worker is compared with the polygon coordinates of the dangerous area, and if the location of the worker is inside the range of the dangerous area polygon, an alarm signal is sent to the worker. This alarm signal is expected to provide workers with detailed information about the expected accidents types. To this end, 14 different alarm signals are provided to represent different types of hazards including falls, dropping, collapse, strangulation, electric shock, landslide, collision, suffocation, fire, overturning, destruction, explosion, and drowning.

\subsection{Data transmission protocol}

Data protocol must be defined for data transmission between each module. In this context, protocol is a standard data format that each module uses to communicate. To establish a protocol, the type of information and the requirement for data processing must be determined. There are two data streams: tag-to-engine; and engine-to-tag. For data transfer from tag to engine, the data protocol includes project ID, tag ID, worker coordinates (i.e. x-y coordinates and floor information) and time of data transmission. On the other hand, for data transfer from engine to tag, the data protocol contains project ID, tag ID, alarm code, alarm number, and interval of alarm output.

The data shown in Tables 5 is formatted as follows. The project ID is used to define each project and is composed in 2 byte form. The tag ID is used to uniquely identify a tag, and hence the worker to whom it is attached, and is composed in 4 byte form. The worker's location is transmitted in the format of two coordinates ( $\mathrm{x}$ and $\mathrm{y}$ ). These coordinates are defined with respect to a building plan datum, and are formatted in "mm" units. Floor information is composed in signature $1 \mathrm{bit}$, and the floor numbering in 7 bits, where 0 marks the ground level and signature 1 marks the basement. Time is composed of 7 bytes, marking year/month/day/hour/minute/second in 2/1/1/1/1/1 byte form. The alarm code expresses warning messages according to warning codes composed in 1 byte form. The alarm number indicates the alarm type and is composed in 1 byte form. Finally, the interval indicates the alarm output frequency (in seconds) and is composed in 1 byte form.

\section{System performance test}

Two case studies were conducted to test the tracking and monitoring performance of the developed systems: 1) an apartment construction site in Yong-In, Korea; and 2) RTLS technology test center at Pusan National Uni- versity in Korea. The performance of tracking can vary subject to the testing environment. For this reason the first case study was conducted to test efficiency and applicability of the system under two different environments: a line of sight (LOS) environment and a non-line of sight (NLOS) environment. An LOS environment is where there are no obstacles like walls, materials, equipment and workers between a tag and a reader. In this environment, signals are well transmitted without any disturbance, so the result is expected to be quite robust of localization performance. On the other hand, in an NLOS environment there are obstacles between a tag and a reader, which can disturb the propagation of the signal by reflection, diffraction, and refraction of the radio waves. This can result in the creation of multipaths, whereby the distance of frequency movement becomes longer than the linear distance. For this reason, in an NLOS environment, tracking performance may be significantly reduced. The basement parking lot was selected for testing an LOS environment and the first three floors of the apartment project were selected for the NLOS environment where walls, columns, and construction equipment act as obstacles in the construction area.

\subsection{Testing under a line of sight environment}

The RTLS hardware (engine, base station, reader, and tag) was installed in the case study apartment site. The personal computer (PC) that hosts the RTLS engine and base stations was installed using a power supply and land-line Local Area Network (LAN). Readers were located in each floor and their coordinates were configured in the engine and a tag was attached to each worker. When workers moved around the site, the deployed RTLS captured their real-time location and represented the information on the 2D site map (displaying each workers' $\mathrm{x}$ and y coordinates) as shown in Figure 5.

After this, the safety manager sets up the location monitoring system. In ArchiCAD 12, the building should be modeled based on existing architectural drawings. The complex, building, and floor were set using building registration function. Building coordinates were created by fixing the referencing point and applying the polyline element. In dangerous zone function, dangerous zone were designated by applying fill element, and the grade of dangerous area is determined according to the risk of work type. Finally, the reference points of equipment and readers' locations are set, and IDs of readers, complex, building, and floor of each reader are set.

Figures 5 and 6 show the monitoring and tracking performance of the developed system under a LOS environment (underground parking lot). As shown in Figure 5 , the system could successfully update the location coordinate of a worker obtained through the RTLS in the

Table 5. Protocol for data transter

\begin{tabular}{c|c|c|c|c|c|c|c}
\hline \multirow{2}{*}{$\begin{array}{c}\text { Data transfer from } \\
\text { tag to engine }\end{array}$} & Data & Project ID & Tag ID & X Coordinate & Y Coordinate & Floor Information & Time \\
\cline { 2 - 7 } & Bytes & 2 & 4 & 4 & 4 & 1 \\
\hline $\begin{array}{c}\text { Data transfer from } \\
\text { engine to tag }\end{array}$ & Data & Project ID & Tag ID & Alarm Code & Alarm Number & Interval \\
\cline { 2 - 8 } & Bytes & 2 & 4 & 1 & 1 & 1 \\
\hline
\end{tabular}




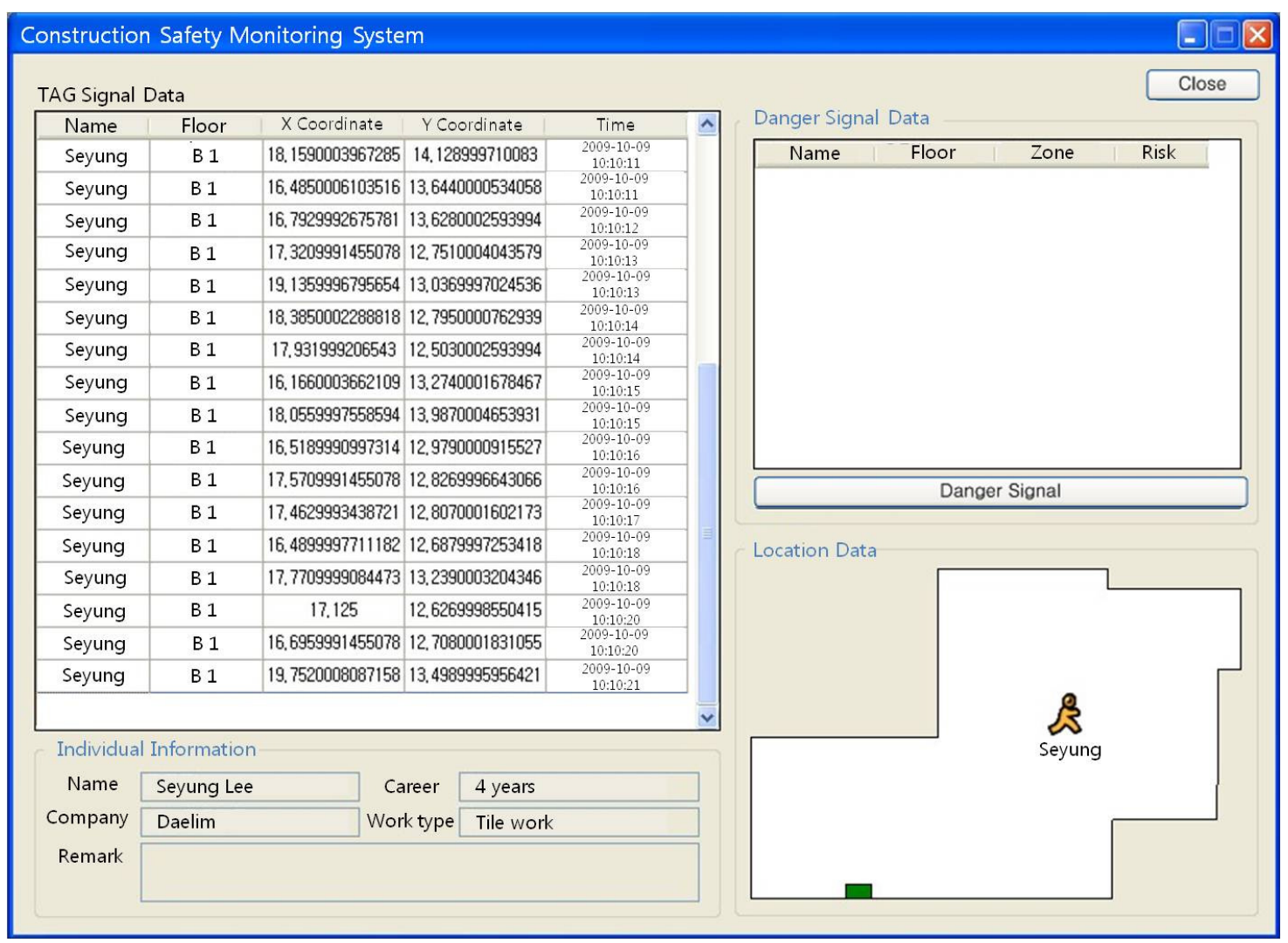

Fig. 5. Monitoring performance under a LOS environment

LOS environment. The 'Danger Signal Data' section represents the entry status for the dangerous areas. Figure 5 indicates that the worker is not in a dangerous area (denoted by green color). The location data is also converted to a visual representation of the building outline, the dangerous areas of the floor and workers' locations. Workers' individual information (e.g. name, career, company, work type, or remark) can also be identified.

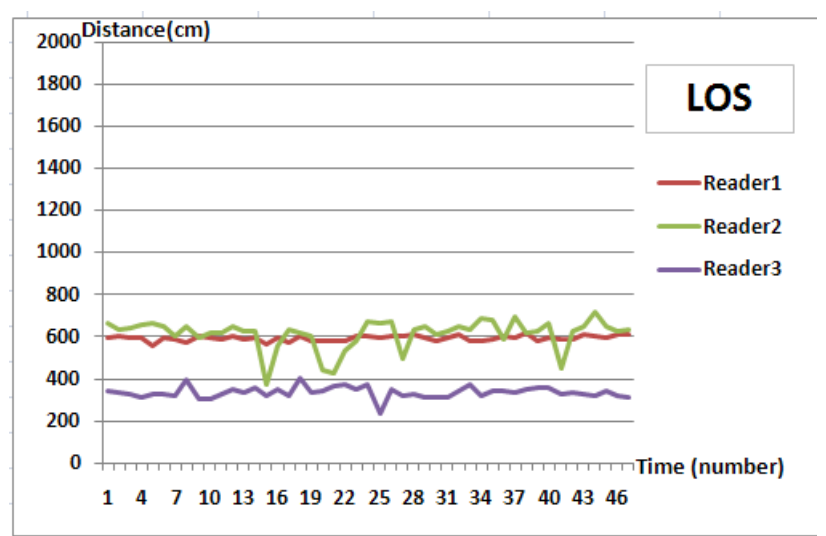

Fig. 6. Tracking performance under a LOS environment

The developed system should be able to measure the tag-to-reader distance for its effective application for the purpose of construction labor safety management. Figure 6 shows the tracking performance of the developed system. Distances between the tag and three readers (Y-axis) are plotted based on each time interval (X-axis).
As shown in Figure 6, the distances between the tag and three readers are relatively consistent in the LOS environment. The average distance between the tag and readers 1 and 2 is about $593 \mathrm{~cm}$ with $12.6 \mathrm{~cm}$ of standard deviation while the average distance between the tag and reader 3 is approximately $336 \mathrm{~cm}$ with $27.4 \mathrm{~cm}$ of standard deviation. Overall, under the LOS environment, the developed system showed an acceptable level of tracking performance, which is attributed to the smooth radio frequency transfer in this environment.

\subsection{Testing under a non-line of sight environment}

The monitoring and tracking performance of the developed system was also tested under an NLOS environment (the first floor). Figure 7 shows the monitoring performance of the developed system in the NLOS environment. In the monitoring system, location data is converted to a visual representation of the location of workers so that the safety manager can identify workers who are in or near the dangerous area and the worker's detailed information. As shown in Figure 7, 'Danger Signal Data' section shows that a worker is approaching a dangerous area. Based on this information, the safety manager can automatically (or manually) send a warning signal to the worker with the detailed information regarding the identified dangerous situation (i.e. dangerous area, worker name, $x-y$ coordinate and floor information, type of danger, and the level of danger). 


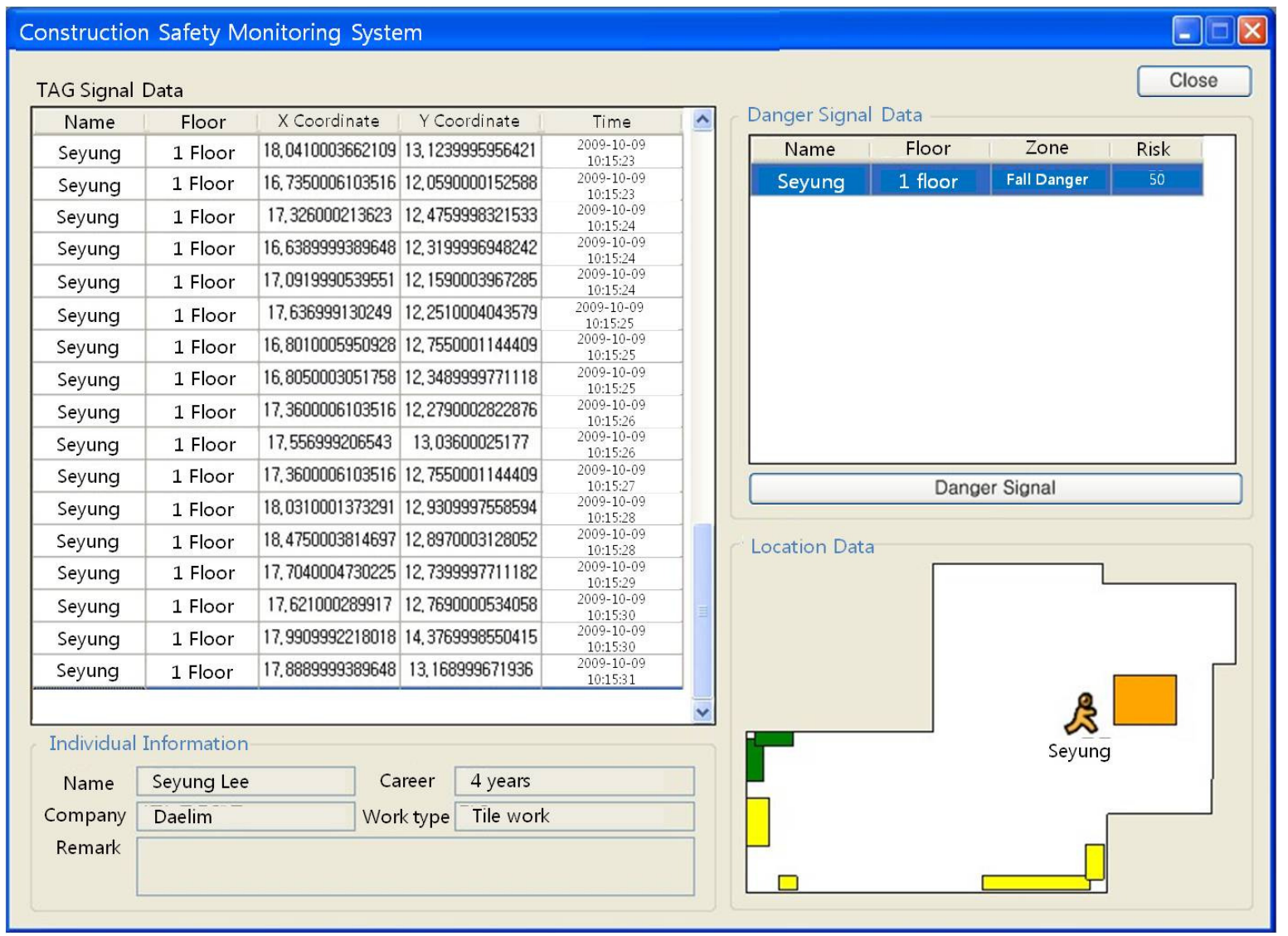

Fig. 7. Monitoring performance under a NLOS environment

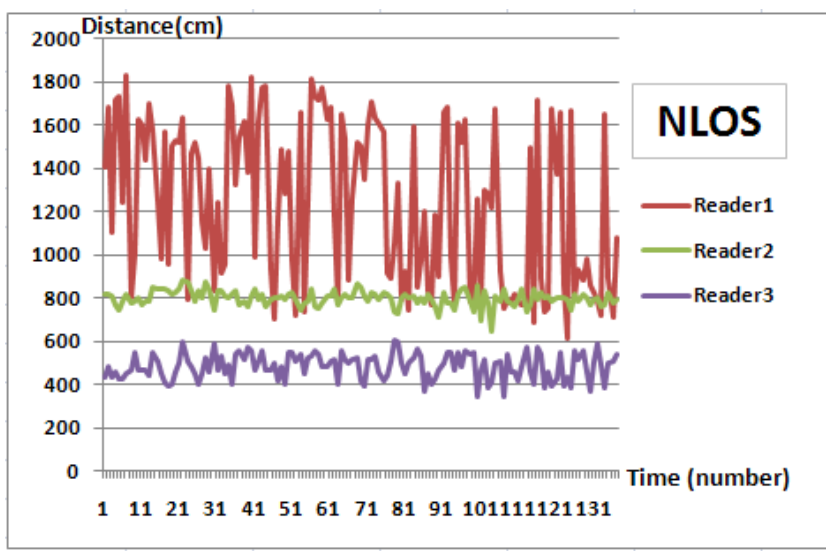

Fig. 8. Tracking performance under a NLOS environment

Figure 8 shows the tracking performance of the developed system in the NLOS environment. As shown in Figure 8, the readers 2 and 3 showed relatively consistent tag-to-reader distance in the NLOS environment. The average distances are $800 \mathrm{~cm}$ and $484 \mathrm{~cm}$ with the $36 \mathrm{~cm}$ and $59 \mathrm{~cm}$ of standard deviation. While these standard deviations are a bit greater than those in the LOS environment, these deviations are acceptable considering the pre-defined threshold to send the early warning signal. However, the distances between the reader 1 and the tag significantly fluctuated widely between $600 \mathrm{~cm}$ and $1,800 \mathrm{~cm}$ with the $371 \mathrm{~cm}$ of standard deviation. This is because there was an obstacle (i.e. structural wall) between the reader 1 and the tag. This testing result confirms that the tracking performance of the developed system can be significantly reduced in an NLOS environment due to signal reflection, refraction, and diffraction and suggests needs for an enhanced tracking performance in an NLOS environment.

\subsection{Application of assistant tag}

The previous test results showed that significant localization errors can exist in the NLOS environment. Construction sites are typically NLOS environments and thus, the localization errors need to be reduced for the developed system to be effectively applied. A time of arrival (TOA)based tracking system requires information concerning the distance between the tag and at least three readers as shown in Figure 2. If the number of readers in a position measures their distance from a given tag is less than three, it is impossible to derive the exact location of the tag using the trilateration method. To address this issue, an assistant tag was applied to increase the tracking performance of the developed system in an NLOS environment. The assistant tag is an additional tag the user can access its location coordinates and this can work as a virtual reader when there is a shortage of readers with line of sight to a particular tag (Cho et al. 2008).

In order to measure the impact of the assistant tag under various obstacles in a safer testing environment, the second case study was conducted in the RTLS technology 
Table 6. Reduction of localization error in an NLOS environment with assistant tag

\begin{tabular}{c|c|c|c|c|c|c}
\hline \multirow{2}{*}{$\begin{array}{c}\text { Type of } \\
\text { obstacle }\end{array}$} & $\begin{array}{c}\text { Actural location } \\
(\mathrm{x}, \mathrm{y})\end{array}$ & \multicolumn{2}{|c|}{ Tracked location } & \multicolumn{3}{|c}{ Localization Error } \\
\cline { 3 - 7 } & & $\begin{array}{c}\text { w/o assis. Tag } \\
(\mathrm{x}, \mathrm{y})\end{array}$ & $\begin{array}{c}\text { w/ assis. Tag } \\
(\mathrm{x}, \mathrm{y})\end{array}$ & $\begin{array}{c}\text { w/o assis. Tag } \\
(\mathrm{cm})\end{array}$ & $\begin{array}{c}\text { w/ assis. Tag } \\
(\mathrm{cm})\end{array}$ & $\begin{array}{c}\text { Reduction } \\
(\%)\end{array}$ \\
\hline Concrete & $(1735,1517)$ & $(2403,972)$ & $(1961,1240)$ & 862 & 357 & 59 \\
\hline Steel & $(2167,289)$ & $(2308,126)$ & $(1816,196)$ & 215 & 123 & 43 \\
\hline Wood & $(3777,1211)$ & $(3504,975)$ & $(3627,1081)$ & 361 & 198 & 45 \\
\hline
\end{tabular}

test center at Pusan National University in Korea, which is very similar to a typical construction site that have many steel obstacles and columns. The test was conducted as follows: first, the global reference point was designated and the readers were located according to the relative distance to the reference point. The location of tags could then be derived based on the location of the readers. In addition, the assistant tags were located nearby the tags which were to be tracked and location coordinates of the assistant tag was stored in the RTLS engine. In order to measure the tracking performance of the developed system, localization error was calculated by comparing the location of the tag obtained from the RTLS and the actual location of the tags, which had previously been measured manually.

Localization Error $(\mathrm{cm})=$

$\sqrt{(\text { Actual } x \text { coordinate }- \text { Tracked } x \text { coordinate })^{2}+(\text { Actual } y \text { coordinate }- \text { Tracked } y \text { coordinate })^{2}}$.

Table 6 shows localization error under three different obstacle types frequently utilized in construction environments including concrete, steel and wood. Also, Table 6 shows that utilization of assistant tag can significantly decrease localization error. For example, when concrete wall served as an obstacle, the localization error without using an assistant tag was $862 \mathrm{~cm}$. However, application of an assistant tag reduced the localization error to $357 \mathrm{~cm}$, which is $59 \%$ of localization error reduction (i.e. $(862-357) / 862)$. The test results indicate that the application of an assistant tag has a great potential to minimize the localization error in NLOS environments. However, these test results also show that further research effort on assistant tag is required to enhance the tracking performance. For example, one research effort required is to optimize the location of assistant tag, which can virtually convert an NLOS environment into an LOS environment. Thus, the authors' subsequent research effort is devoted to the development of an optimization algorithm to improve localization performance. Interested readers can find more details regarding the use of assistant tag from Lee et al. (2012).

\section{Conclusions}

Every accident has its causes and can be prevented by identifying and eliminating the causes. According to Heinrich (1959), the causes of an accident can be classified into indirect accident causes (i.e. deficiencies in safety management) and direct causes of accidents (e.g. unsafe mechanical or physical conditions and unsafe acts). Direct causes of accidents are unique in each individual construction site. Based on this recognition, this study introduced a real-time location-based construction labor safety management system. For this purpose, a real-time locating system (RTLS) was applied to track location of workers on a real-time basis, and a location monitoring system was developed where workers' locations and potential dangerous areas are visualized. Finally, an alarming technology was utilized to warn endangered workers. Integrating these technologies, the developed system can assist the management and control of direct causes of accidents in real-time. Two case studies were conducted in order to test the tracking and monitoring performance of the developed system. The case studies confirmed that the developed system can show an acceptable tracking and monitoring performance in a LOS environment; however, exhibit a significant localization error which should be overcame in order for the system to be utilized for the purpose of construction safety management. To address this issue, this paper adopted assistant tags and the case study confirmed that application of an assistant tag has a great potential to decrease the localization error in an NLOS environment and increase the applicability of the developed construction labor safety system.

While the developed system was proved to be effective to prevent construction accident, it also has several limitations that should be overcome in the following studies. First, the threshold value of dangerous zones need to be further investigated by considering the moving velocity of a worker or an object and the required time for a worker to respond to the identified danger after receiving warning signals. Also, the developed system needs to be tested under large number of workers. Lastly, identification and registration of accident risk needs to be automated. Overcoming these limitations would enhance the performance and applicability of the construction labor safety management system and ultimately minimize construction accidents and hazards.

\section{References}

Behzadan, A. H.; Kamat, V. R. 2009. Automated generation of operations level construction animations in outdoor augmented reality, Journal of Computing in Civil Engineering ASCE 23(6): 405-417. http://dx.doi.org/10.1061/(ASCE)0887-3801(2009)23:6(405)

Carbonari, A.; Giretti, A.; Naticchia, B. 2011. A proactive system for real-time safety management in construction sites, Automation in Construction 20(6): 686-698. http://dx.doi.org/10.1016/j.autcon.2011.04.019

Chae, S.; Yoshida, T. 2010. Application of RFID technology to prevention of collision accident with heavy equipment, Automation in Construction 19(3): 368-374. http://dx.doi.org/10.1016/j.autcon.2009.12.008 
Cheng, T.; Teizer, J. 2010. Real-time data collection and visualization technology in construction, in Proc. of the Construction Research Congress, ASCE, Reston, 339348. http://dx.doi.org/10.1061/41109(373)34

Chi, S.; Caldas, C. 2012. Image-based safety assessment: automated spatial safety risk identification of earthmoving and surface mining activities, Journal of Construction Engineering and Management ASCE 138(3): 341-351. http://dx.doi.org/10.1061/(ASCE)CO.1943-7862.0000438

Chi, S.; Caldas, C. 2011. Automated object identification using optical video cameras on construction sites, ComputerAided Civil and Infrastructure Engineering 26(5): 368380. http://dx.doi.org/10.1111/j.1467-8667.2010.00690.x

Chin, S.; Yoon, S.; Choi, C.; Cho, C. 2008. RFID + 4D for progress management of structural steel works in high-rise building, Journal of Computing in Civil Engineering ASCE 22(2): 74-89. http://dx.doi.org/10.1061/(ASCE)0887-3801(2008)22:2(74)

Cho, H.; Jung, Y.; Choi, H.; Jang, H.; Son, S.; Baek, Y. 2008. Precise location tracking system based on time difference of arrival over LR-WPAN, International Conference on Mobile Computing and Networking, 14-19 September 2008, San Francisco, California, USA, 67-72. http://dx.doi.org/10.1145/1410012.1410028

Dai, F.; Lu, M.; Kamat, V. R. 2011. Analytical Approach to Augmenting Site Photos with 3D Graphics of Underground Infrastructure in Constrution Engineering Applications, Journal of Computing in Civil Engineering ASCE 25(1): 66-74. http://dx.doi.org/10.1061/(ASCE)CP.1943-5487.0000072

Dziadak, K.; Kumar, B.; Sommerville, J. 2009. Model for the 3D location of buried assets based on RFID technology, Journal of Computing in Civil Engineering ASCE 23(3): $148-159$.

http://dx.doi.org/10.1061/(ASCE)0887-3801(2009)23:3(148)

Ergen, E.; Akinci, B.; East, B.; Kirby, J. 2007. Tracking components and maintenance history within a facility utilizing radio frequency identification technology, Journal of Computing in Civil Engineering ASCE 21(1): 11-20.

http://dx.doi.org/10.1061/(ASCE)0887-3801(2007)21:1(11)

Fosbroke, D. E. 2004. Studies on heavy equipment blind spots and internal traffic control, in Proc. of the $3^{\text {rd }}$ Int. Roadway Construction Safety and Health Conference, National Institute for Occupational Safety and Health, Atlanta, 31-40.

Fullerton, C. E.; Allread, B. S.; Teizer, J. 2009. Pro-active-realtime personnel warning system, in Proc. of the Construction Research Congress, 5-7 April 2009, ASCE, Reston, 31-40. http://dx.doi.org/10.1061/41020(339)4

Golparvar-Fard, M.; Pena-Mora, F.; Arboleda, C. A.; Lee, S. 2009. Visualization of construction progress monitoring with 4D simulation model overlaid on time-lapsed photograghs, Journal of Computing in Civil Engineering ASCE 23(6): 391-404.

http://dx.doi.org/10.1061/(ASCE)0887-3801(2009)23:6(391)

GRAPHISOFT 2008. Available from Internet: http://www.graphisoft.com/

Hallowell, M. R.; Teizer, J.; Blaney, W. 2010. Application of sensing technology to safety management, in Proc. of the Construction Research Congress, 8-10 May 2010, ASCE, Reston, 31-40. http://dx.doi.org/10.1061/41109(373)4
Heesom, D.; Mahdjoubi, L.; Proverbs, D. 2003. A dynamic VR system for visualizing construction space usage, Construction Research: 1-8. http://dx.doi.org/10.1061/40671(2003)117

Heinrich, H. W. 1959. Industrial accident prevention: a scientific approach. NewYork: McGraw-Hill Book Company.

Hinze, J.; Pedersen, C.; Fredley, J. 1998. Identifying root causes of construction injuries, Journal of Construction Engineering and Management ASCE 124(1): 67-71. http://dx.doi.org/10.1061/(ASCE)07339364(1998)124:1(67)

Irizarry, J.; Abraham, D. M. 2005. Application of virtual reality technology for the improvement of safety in the steel erection process, Computing in Civil Engineering, 1-11. http://dx.doi.org/10.1061/40794(179)148

Issa, R. R. A. 2000. Virtual reality: a solution to seamless technology integration in the AEC industry, Cosntruction Congress, 20-22 February 2000, Orlando, Florida, USA, 1007-1013. http://dx.doi.org/10.1061/40475(278)107

Jaselskis, E. J.; EI-Misalami, T. 2003. Implementing radio frequency identification in the construction process, Journal of Construction Engineering and Management ASCE 129(6): 680-688. http://dx.doi.org/10.1061/(ASCE)07339364(2003)129:6(680)

Jing, Z.; Dianhui, Z.; Dongyuan, Y. 2008. The people flow and the logistics managing system at expo based on RFID and 2-D code technology, in The Eighth International Conference of Chinese Logistics and Transportation Professionals, July 31 - August 3, 2008, Chengdu, China, 1941-1948. http://dx.doi.org/10.1061/40996(330)285

KOSHA (Korea Occupational Safety and Health Agency). 2007. The risk assessment model by work type in construction industry: Research Report (2009-174-755), $12-24$.

Lee, H.; Lee, K.; Park, M.; Baek, Y.; Lee, S. 2012. RFID-based real-time locating system for construction safety management, Journal of Computing in Civil Engineering ASCE 26(3): 366-377. http://dx.doi.org/10.1061/(ASCE)CP.1943-5487.0000144

Navon, R.; Kolton, O. 2007. Algorithms for automated monitoring and control of fall hazards, Journal of Computing in Civil Engineering ASCE 21(1): 21-28. http://dx.doi.org/10.1061/(ASCE)08873801(2007)21:1(21)

Reese, C.; Eidson, J. 2006. Handbook of Osha construction safety and health. $2^{\text {nd }}$ ed. New York: Taylor \& Francis.

Son, S.; Kim, J.; Choi, H.; Jung, Y.; Baek, Y. 2009. Demo abstract: implementation of RTLS to overcome radio shadow area, in Fifteenth IEEE Real-Time and Embedded Technology and Applicationi Sysmposium.

Song, J.; Hass, C. T.; Caldas, C. H. 2006. Tracking the location of materials on construction job sites, Journal of Construction Engineering and Management ASCE 132(9): 911-918. http://dx.doi.org/10.1061/(ASCE)07339364(2006)132:9(911)

Tarawneh, M. S. 2001. Evaluation of pedestrian speed in Jordan with investigation of some contributing factors, Journal of Safety Research 32(2): 229-236. http://dx.doi.org/10.1016/S0022-4375(01)00046-9

Teizer, J.; Castro-Lacouture, D. 2007. Combined ultrawideband positioning and range imaging sensing for productivity and safety monitoring in building 
construction, in Proc. of the 2007 ASCE Int. Workshop on Computing in Engineering, ASCE, Reston, 681-688. http://dx.doi.org/10.1061/40937(261)81

US Bureau of Labor. 2006 [online], [cited 18 December 2009]. Available from Internet: http://www.bls.gov/lif/ 2006
Wu, W.; Yang, H.; Chew, D. A. S.; Yang, S.; Gibb, A. G. F.; Li, Q. 2010. Towards an autonomous real-time tracking system of nearmiss accidents on construction sites, Automation in Construction 19(2): 134-141. http://dx.doi.org/10.1016/j.autcon.2009.11.017

Kwang-Pyo LEE is a PhD candidate in the Department of Architecture at Seoul National University, Seoul, Korea. He is a member of Korea Institute of Construction Engineering and Management and Architectural Institute of Korea. His research interests include construction IT, construction safety, and construction management.

Hyun-Soo LEE is a Professor at the Department of Architecture at Seoul National University, Seoul, Korea. He served as the Chairman of the Korea Institute of Construction Engineering and Management. His research interests include cost engineering, career development program and construction policy.

Moonseo PARK is a Professor at the Department of Architecture at Seoul National University, Seoul, Koera. He served as Associate Editor for the ASCE Journal of Construction Engineering and Management, and the ASCE Journal of Computing in Civil Engineering. His research interests include simulation modeling, construction IT, construction safety and governmental policies.

Hyunsoo KIM is a PhD candidate in the Department of Architecture at Seoul National University, Seoul, Korea. He is a member of Korean Institute of Construction Engineering and Management and Architectural Institute of Korea. His research interests include construction IT, construction safety and construction productivity.

Sangwon HAN is an Assistant Professor in the Department of Architectural Engineering at the University of Seoul, Seoul, Korea. He also worked as a Lecturer at the University of New South Wales, Sydney, Australia. His research interests include construction productivity improvement, computer simulation modeling and information technology in construction. 